\title{
Our Experience with Hyperosmolar Treatment for Patients with Traumatic Brain Injury in a Pediatric Intensive Care Unit
}

\author{
Abdullah Yazar, Esra Türe, Fatih Akın \\ Necmettin Erbakan University, Meram Faculty of Medicine, Konya, Turkey
}

\begin{abstract}
Introduction: Trauma that occurs during childhood is a leading preventable health problem worldwide. The aim of this study was to compare the epidemiological characteristics, trauma etiologies, treatment procedures, efficacy, and side effects of $3 \%$ hypertonic saline and mannitol treatment for patients in a pediatric intensive care unit with the diagnosis of traumatic brain injury (TBI) or brain edema.

Methods: The files of 35 patients with the diagnosis of TBI or brain edema were reviewed retrospectively. Patients were divided into 3 groups according to the brain edema treatment procedure applied. Group 1 comprised patients who received only 3\% hypertonic saline, patients who received both hypertonic saline and mannitol were included in Group 2, and Group 3 was made up of those who received only mannitol. Patients were also categorized according to their admission Glasgow Coma Score (GCS)

Results: Sixteen (45.7\%) of the 35 patients were female and 19 (54.3\%) were male. An evaluation of complications that developed in the course of treatment revealed that renal failure was the most frequent, observed in 17 patients (48.6\%). Of the 17 patients who developed renal failure, 14 (82.4\%) were evaluated as severe TBI based on GCS, which was statistically significant $(p=0.004)$. When the brain edema treatment of these 17 patients was examined, there was statistical significance in the finding that $15(88.2 \%)$ of the patients had received mannitol ( $p=0.037)$. The serum urea and creatinine levels of patients who developed renal failure were found to be positively correlated with the number of mannitol doses received $(r=0.784 ; p=0.001)$. Discussion and Conclusion: Hypertonic saline alone or in combination with mannitol is used in the treatment of brain edema. The present findings suggest that 3\% hypertonic saline is safer in hyperosmolar treatment of traumatic brain injury and that mannitol should be used with caution, especially in young patients. Further studies evaluating the efficacy and side effects of hyperosmolar treatment procedures are needed, especially in the childhood age group.
\end{abstract}

Keywords: 3\% hypertonic saline; brain edema; mannitol.

A $s$ one of the leading causes of trauma-related deaths, traumatic brain injury (TBI) is an important health problem that seriously affects young people worldwide; it frequently results in neurological sequelae, and it requires a multidisciplinary approach ${ }^{[1]}$. Brain edema in children who had a serious cerebral injury increases intracranial pressure, subsequently decreases cerebral perfusion pressure, and induces development of secondary ischemic brain injury ${ }^{[2]}$. A Glasgow Coma Score (GCS) of $\leq 8$ points is defined as severe $\mathrm{TBI}$ and is associated with a poor prognosis and a higher mortality rate, requiring aggressive management ${ }^{[3]}$. Hyperosmolar therapy (20\% mannitol or $3 \%$ hypertonic saline)

Correspondence (iletişim): Abdullah Yazar, M.D. Necmettin Erbakan University, Meram Faculty of Medicine, Konya, Turkey Phone (Telefon): +90332 2236593 E-mail (E-posta): drabdullahyazar@hotmail.com

Submitted Date (Başvuru Tarihi): 17.11.2017 Accepted Date(Kabul Tarihi): 11.12.2017 
is a first-line treatment in the management of intracranial hypertension.

The objective of this study was to analyze the epidemiological characteristics and the etiologies of trauma in pediatric patients who developed brain edema secondary to $\mathrm{TBI}$, the treatment they received, the effectiveness, and side effects of hypertonic saline and mannitol therapies administered in a pediatric intensive care unit.

\section{Materials and Methods}

The medical records and admission files of 35 moderate/ severe TBI patients under the age of 18 years who were followed up in the pediatric intensive care unit (ICU) with the indication of brain edema between September 2015 and September 2017 were retrospectively analyzed. Prior to initiating the study, approval was granted by the Ethics Committee. Gender, age (years), the type of traumatic event, admission GCS, and test results were recorded. The laboratory test results used were pre- and post-treatment hemoglobin count $(\mathrm{g} / \mathrm{dL})$, hematocrit $(\%)$, platelet count $\left(10^{3} / \mathrm{uL}\right)$, urea level (mg/dL), creatinine level ( $\mathrm{mg} / \mathrm{dL}$ ), osmolarity (mOsmol/L), and coagulation parameters, as well as radiological imaging results, length of stay in the ICU, mechanical ventilator support details, data of treatment provided ( $3 \%$ hypertonic saline, mannitol, antiepileptic treatment, sodium-thiopental), additional drug dosages, and post-treatment complications. The patients were divided into 3 groups based on their admission GCS TBI score of mild (GCS: 14-15), moderate (GCS: 9-13), or severe (GKS $\leq 8$ ). Mild cases were not included in the study. The patients were then divided into groups based on the treatment they received: Group 1 was given only hypertonic saline, Group 2 received hypertonic saline and mannitol, and Group 3 was treated with mannitol alone. In order to maintain serum sodium values between $155-160 \mathrm{mEq} / \mathrm{L}$, $3 \%$ hypertonic saline was administered. The first intravenous loading dose was $5 \mathrm{~mL} / \mathrm{kg}$, and recurrent doses were adjusted based on serum sodium level. A maintenance dose of $3 \%$ hypertonic saline was given in hourly doses of 0.5 to 1.5 $\mathrm{mL} / \mathrm{kg}$. Serum osmolarity was maintained at approximately $360 \mathrm{mOsmol} / \mathrm{L}$ in patients who received only 3\% hypertonic saline treatment. Mannitol was administered at 6-hour intervals in doses of 0.25 to $0.5 \mathrm{~g} / \mathrm{kg}$. Serum electrolytes and osmolarity levels were checked at 4-hour intervals, and the osmolar gap was calculated. The intent was to keep the osmolarity level between 310-320 mOsmol/L, and mannitol treatment was discontinued when serum osmolarity was above $320 \mathrm{mOsmol} / \mathrm{L}$. The osmolar gap was estimated to follow the mannitol clearance, and it was maintained at less than $20 \mathrm{mOsm} / \mathrm{kg}$. In patients who received 3\% hypertonic saline and mannitol solution, mannitol treatment was stopped and 3\% hypertonic saline treatment was maintained when osmolarity reached $320 \mathrm{mOsmol} / \mathrm{L}$. Patients with renal failure and active intracranial bleeding were not given mannitol treatment. All patients with severe TBI were given sodium thiopental (loading dose of $5-10 \mathrm{mg} / \mathrm{kg}$ followed by maintenance dose of 3-5 mg/kg/hour) to induce barbiturate coma. All patients under barbiturate treatment were followed closely for side effects, such as hypotension and cardiac suppression, and invasive blood pressure monitoring was performed. Continuous electroencephalographic monitoring was also performed in order to adjust the drug dose to the lowest that would provide a burst-suppression pattern. Body temperature, pulse rate, blood pressure, central venous pressure, oxygen saturation, fluid-electrolyte balance, and urine output of all patients were monitored. Hypothermia was induced in all patients, and body temperature was maintained below $360 \mathrm{C}$. Therapeutic ventilation to maintain partial carbon dioxide levels between 30 and 35 $\mathrm{mmHg}$ was applied. The patients' heads were maintained in a midline position, and bedheads were elevated to between 150 and 300 . Prophylactic doses of antiepileptics (phenytoin, phenobarbital, levetiracetam) were initiated. Midazolam and a fentanyl infusion were given to achieve sedation and analgesia. Brain edema and intracranial pressure (ICP) were monitored both clinically and radiologically.

Statistical analysis was performed using IBM SPSS Statistics for Windows, Version 20.0 (IBM Corp., Armonk, NY, USA). Descriptive statistics were used to analyze the distribution and frequency of the data, and a chi-square test was used to compare frequency in independent groups. The normality of distribution of continuous variables was examined. Pearson correlation analysis was performed for data with a normal distribution pattern, and Spearman correlation analysis was used for data with non-normal distribution, as well as for ordinal data. For all statistical analyses, the level of statistical significant was $p<0.05$.

\section{Results}

A total of 35 patients (female: $n=16,45.7 \%$; male: $n=19$, $54.3 \%$ ) were treated for brain edema. The median age was 4.20 years (min: 0.50, max: 14.10 years). Among females, the median age was 3.52 years (min: 0.50 years, max: 9.45 years), and among males the median age was 5.20 years (min: 0.76, max: 14.10 years). Epidemiological characteristics of the patients are provided in Table 1. A statistically significant difference was not detected with regard to gender, patient age, or treatment method $(p>0.05)$. The severity of 


\begin{tabular}{|c|c|c|c|c|}
\hline Characteristics & $\begin{array}{l}\text { Hypertonic saline } \\
\qquad(n=10)\end{array}$ & $\begin{array}{l}\text { Hypertonic saline } \\
\text { and mannitol } \\
(n=16)\end{array}$ & $\begin{array}{c}\text { Mannitol } \\
(n=9)\end{array}$ & $\begin{array}{l}\text { Total } \\
(n=35)\end{array}$ \\
\hline \multirow[t]{2}{*}{ Age (years) } & 2.85 & 3.35 & 5.12 & 4.20 \\
\hline & $(0.62-12.20)$ & $(0.50-14.10)$ & $(3.25-9.45)$ & $(0.50-14.10)$ \\
\hline Female $(n, \%)$ & $6.60 \%$ & 5. $32.2 \%$ & $5.55 .6 \%$ & $16.45 .7 \%$ \\
\hline Male $(n, \%)$ & 4. $40 \%$ & $11.68 .8 \%$ & 4. $44.4 \%$ & $19.54 .3 \%$ \\
\hline GCS (mean) & $7.80 \pm 2.65$ & $6.18 \pm 3.22$ & $8.77 \pm 1.64$ & $7.31 \pm 2.88$ \\
\hline \multicolumn{5}{|l|}{ Mechanism of trauma } \\
\hline In-vehicle accident & 4. $40 \%$ & 6. $37.4 \%$ & $5.55 .6 \%$ & $15.42 .9 \%$ \\
\hline Other vehicle accident & 4. $40 \%$ & 4. $25 \%$ & 4. $44.4 \%$ & 12. $34.3 \%$ \\
\hline Fall from a height & 2. $20 \%$ & 3. $18.8 \%$ & $0.0 \%$ & 5. $14.3 \%$ \\
\hline Drowning & $0.0 \%$ & 3. $18.8 \%$ & $0.0 \%$ & 3. $8.5 \%$ \\
\hline
\end{tabular}

GCS: Glasgow coma score.

TBI was analyzed according to age, and it was found that in the severe TBI group, 12 patients (60\%) were under 5 years of age, and in the moderate TBI group, 8 patients (53.3\%) were under 5 years of age. No statistically significant difference was detected between age group and the severity of the trauma ( $p>0.05)$. However, 11 of 15 (73.3\%) victims of in-vehicle accidents were female, which was statistically significant $(p=0.026)$.

Only $2(5.7 \%)$ of the study patients were admitted to the hospital less than 15 minutes after the trauma, while 19 (54.3\%) patients arrived within 16 to 30 minutes. A statistically significant difference was not detected between the length of time until arrival at the hospital and the etiology of the trauma ( $p>0.05)$. The GCS score of patients at admission was $\leq 8$ points in 20 (57.1\%) and between 9 and 13 in 15 (42.9\%) patients. No statistically significant difference was detected between gender, age group, etiology of the trauma, and GCS score ( $p>0.05)$. A statistically significant finding was that all 7 of the patients with fixed and dilated pupils ( $p=0.005)$, and the 10 who did not display a light reflex $(p=0.002)$ were in the severe TBI group. In addition, it was also statistically significant that all $6(17.1 \%)$ patients who received more than 10 doses of mannitol were in the severe TBI group ( $p=0.017)$.

The patients were also evaluated based on the presence of complications. Fifteen (42.9\%) patients were discharged without sequelae, while 6 (17.1\%) patients exited. There was the development of hypoxic ischemic encephalopathy in $9(25.7 \%)$, cerebral salt-wasting in $3(8.6 \%)$, inappropriate antidiuretic hormone secretion syndrome in $1(2.9 \%)$, and hemiplegia in 1 (2.9\%) patient. Most (57.1\%) of these complications were seen in the severe TBI group. All of the patients who died were in the severe TBI group, which was a statistically significant finding $(p=0.016)$. Though not statistically significant, 5 of the 6 fatalities were in Group 2 , which received both hypertonic saline and mannitol $(p>0,05)$. Additionally, renal failure was seen in 17 (48.6\%) patients. Fourteen (82.4\%) of the 17 patients who developed renal failure were in the severe TBI group, which was statistically significant $(p=0.004)$. A statistically significant number of patients $(15 / 17,88.2 \%)$ who developed renal failure received mannitol treatment $(p=0.037)$. A positive correlation was seen in patients who developed renal failure between the serum urea and creatinine values and the number of mannitol doses $(r=0.784 ; p=0.001)$. Renal failure developed in only 2 patients who received hypertonic saline treatment. Most of the patients who developed renal failure $(11 / 17,64.7 \%)$ were younger than 5 years of age, though it was not statistically significant $(p>0.05)$. Osmolarity and renal function test values of the patients according to treatment group can be seen in Table 2 .

Admission computed tomography images revealed brain edema in $28.6 \%(n=10)$ patients. In addition to brain edema, subarachnoid bleeding and compression fracture were detected in $25.7 \%$ and $17.1 \%$, respectively (Fig. 1). A decompressive craniectomy was performed in only 1 case. ICP monitoring was not performed in any patient. A total of $27(77.1 \%)$ patients required mechanical ventilation, and a statistically significant number of them $(n=20 ; 74.1 \%)$ were in the severe TBI group $(p=0.001)$. The mean length of ICU stay was $11.22 \pm 7.32$ days (min: 2 days, max: 32 days). A positive, but statistically insignificant correlation was seen between the length of stay in the ICU and mortality $(p>0.05)$. A statistically significant number $(n=19,79.2 \%)$ of patients among those who received barbiturate treat- 


\begin{tabular}{|c|c|c|c|c|}
\hline Pre-treatment osmolarity (mOsm/L) & $284.30 \pm 6.49$ & $283.93 \pm 7.30$ & $279.44 \pm 1.66$ & $>0.05$ \\
\hline Post-treatment osmolarity (mOsm/L) & $344.80 \pm 7.09$ & $329.06 \pm 2.71$ & $320.66 \pm 3.04$ & $>0.05$ \\
\hline Pre-treatment creatinine (mg/dL) & $0.95 \pm 0.08$ & $0.78 \pm 0.06$ & $0.89 \pm 0.04$ & $>0.05$ \\
\hline Post-treatment creatinine (mg/dL) & $1.11 \pm 0.27$ & $1.70 \pm 0.66$ & $1.65 \pm 0.57$ & 0.001 \\
\hline
\end{tabular}

BUN: Blood urea nitrogen.

ment $(n=24,68.6 \%)$ were in the severe TBI group $(p=0.001)$. The remaining 5 patients were in the moderate TBI group; however, barbiturate treatment was initiated when the brain edema worsened. Though only 11 (31.4\%) patients experienced seizures, antiepileptic treatment was given to all patients as a prophylactic. Seven (63.6\%) patients who experienced seizures were in the severe TBI group, but it was not statistically significant $(p>0.05)$. The antiepileptics most frequently administered were phenobarbital (45.7\%) and phenytoin (45.7\%), and in some cases, levetiracetam (8.6\%).

\section{Discussion}

TBI is one of the leading causes of pediatric trauma mortality; it affects between 47 and 280 of every 100,000 children worldwide ${ }^{[4]}$. Generally, a motor vehicle accident or a fall from a height is the cause of most of these injuries ${ }^{[5]}$. In the present study, the most frequent cause of injury was a traffic accident (77.1\%). The severity of a TBI is defined using the GCS or the pediatric GCS, and it is classified as

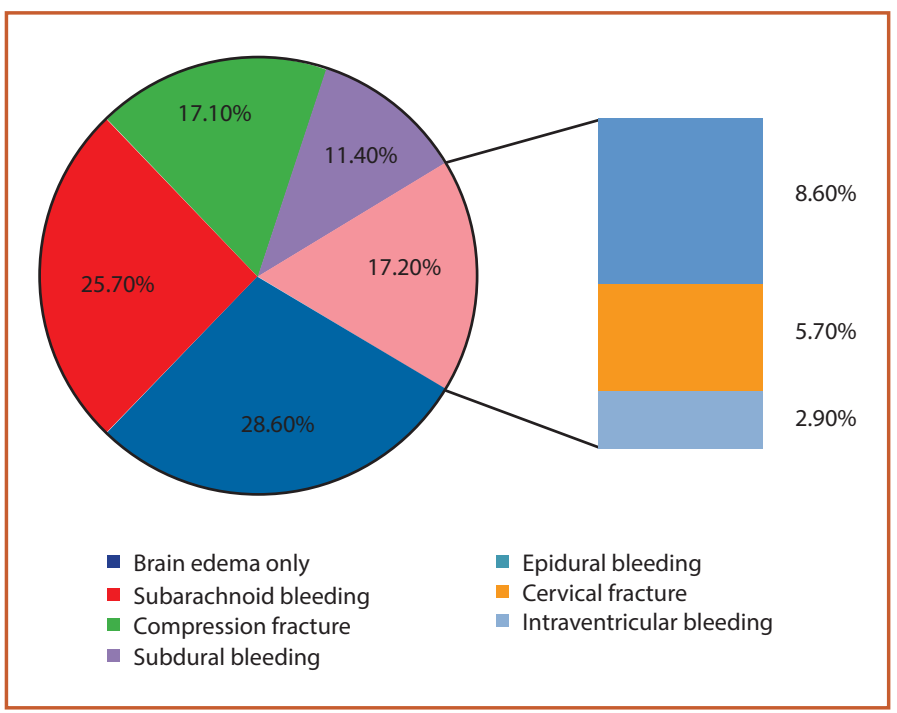

Figure 1. Computed tomography findings of the patients at admission. mild, moderate, or severe ${ }^{[6]}$. Most pediatric trauma patients seen in the emergency room are mild cases. Severe $\mathrm{TBI}$, defined as a GCS $\leq 8$ points, has a poor prognosis and a high mortality rate ${ }^{[7]}$. The highest morbidity and mortality rates have been reported in children younger than 4 years of age and those with lower GCS scores ${ }^{[8]}$. In our study, too, though not statistically significant, $50 \%$ of those who died were younger than 5 years of age, and it was a statistically significant finding that all of those who exited were in the severe TBI group $(p=0.016)$.

A series of biochemical, cellular, and metabolic responses to the damage caused by direct primary cerebral trauma or secondary to brain injury induced by hypoxia and/or hypotension play a role in the pathophysiology of TBI [9-11]. An increase in ICP caused by brain damage can be detected using brain imaging or other noninvasive methods, accompanied by clinical findings. Invasive ICP measurement with an external ventricular drain or an intraparenchymal ICP monitor definitively reveals the presence of intracranial hypertension ( $>20 \mathrm{mmHg}$ ). These measurements are performed in cases of severe TBI following head trauma with a GCS score $\leq 8$ and potential complications, such as infection or bleeding ${ }^{[12,13]}$. We could not perform invasive ICP monitoring on any of our patients due to technical limitations. Brain edema and changes in ICP were followed up using clinical and radiological methods. A decompressive craniectomy was performed on only 1 patient because of a rapid deterioration in clinical status and an increase in brain edema detected during radiological examination. Invasive ICP monitoring aids in treatment planning and the decision whether or not a decompressive craniectomy is required. If the necessary facilities are available, a decompressive craniectomy should be performed in cases of severe TBI.

Hyperosmolar therapy (20\% mannitol or $3 \%$ hypertonic saline) is the first-line treatment in the management of intracranial hypertension. The mechanism of action of these substances involves decreasing blood viscosity and the 
oncotic movement of water into the intravascular compartment, leading to a reduction in cerebral intracellular fluid. ${ }^{[14]}$. The best osmolar therapy regimen for children with increased ICP has not been confirmed. In most pediatric patients, osmolar therapy with mannitol or hypertonic saline is effective as an initial treatment ${ }^{[12]}$. When used in combination, hypertonic saline aids in balancing hyponatremia and hypovolemia following rapid diuresis related to mannitol treatment. Mannitol helps to compensate for aggravation of vasogenic cerebral edema that might emerge after long-term use of hypertonic saline. Hypertonic saline or mannitol forms an osmotic gradient, which induces the outflow of free water into the extracellular space and into circulation, and thereby leads to a decrease in intracranial fluid volume and ICP [15].

There is limited evidence concerning the superiority of mannitol or hypertonic saline in the management of intracranial hypertension. Since mannitol can decrease ICP more rapidly, some specialists prefer it for the acute treatment of children presenting with acute head trauma and in cases of herniation, while others prefer to use hypertonic saline. [16]. Mannitol should not be given as a continuous infusion ${ }^{[17]}$. It may be administered in 4-hour intervals. Before administration of each dose of mannitol, serum osmolarity should be evaluated. Use of mannitol is contraindicated in patients with active intracranial bleeding ${ }^{[12]}$. Mannitol therapy also has the potential side effects of hyperosmolarity, hypovolemia, electrolyte imbalance, and acute renal failure. These adverse effects are more frequently seen with chronic administration or higher doses. Patients receiving mannitol for a long period of time or at high doses should be closely monitored. Serum osmolarity, electrolyte balance, and renal function should be evaluated at least every 6 to 8 hours, and before the next dose. In addition, high-dose or long-term use of mannitol can lead to a rebound increase in ICP if the mannitol passes through blood-brain barrier ${ }^{[17]}$. Serum osmolarity should be maintained at 300 to $320 \mathrm{mOsm} / \mathrm{kg}$ in patients receiving mannitol therapy. Mannitol induces osmotic diuresis; therefore, a urethral Foley catheter should be inserted to prevent overdistention of the bladder and to monitor urine output ${ }^{[12]}$. In our study, consistent with the literature, a statistically significant number of our patients $(15 / 17 ; 88.2 \%)$ who developed renal failure had received mannitol treatment $(p=0.037)$. A positive correlation was seen between the number of mannitol doses and serum urea and creatinine levels in patients who developed renal failure $(p=0.001)$. Patients with renal failure were evaluated according to age group, and though not statistically significant, 11 of 17 (64.7\%) patients who developed renal failure were younger than 5 years of age ( $p>0.05$ ). This finding suggests that when planning hyperosmolar treatment for TBI, particularly in young patients, mannitol should be used with care, and if possible, the use of hypertonic solution should be preferred.

There is little research concerning the optimal dose of hypertonic saline for the medical treatment of intracranial blood pressure, the concentration to be used, or the indications for mannitol versus hypertonic saline ${ }^{[16]}$. An initial $3 \%$ hypertonic saline bolus of $5 \mathrm{~mL} / \mathrm{kg}$ corresponds to an increase of $5 \mathrm{mEq} / \mathrm{L}$ in serum sodium. This dose may be repeated at hourly intervals until the serum sodium level reaches $160 \mathrm{mEq} / \mathrm{L}$. Typically, when the serum sodium level exceeds $160 \mathrm{mEq} / \mathrm{L}$, 3\% hypertonic saline solution does not further decrease ICP. An hourly dose of 0.5 to $1.5 \mathrm{~mL} / \mathrm{kg}$ of $3 \%$ hypertonic saline to maintain ICP below $20 \mathrm{mmHg}$ may be administered following control of ICP with bolus doses $[12,18]$. Unlike mannitol, $3 \%$ hypertonic saline solution does not induce profound osmotic diuresis, and the risk of hypovolemic complications is lower. Furthermore, it has the additional beneficial effects of restoration of normal cellular resting potential and cell volume, stimulation of the release of atrial natriuretic peptide, prevention of inflammation, and increased cardiac output. Potential complications related to $3 \%$ hypertonic saline administration include hypernatremia, hyperosmolarity, acute renal injury, pulmonary edema and/or heart failure, metabolic acidosis, osmotic demineralization syndrome, and fluid overload ${ }^{[12,17]}$. In the present study, fewer side effects were observed in patients who received $3 \%$ hypertonic saline compared with mannitol. In pediatric patients with TBI, $3 \%$ hypertonic saline solution can be used safely.

Barbiturates are used to treat intracranial hypertension refractory to other methods. Pentobarbital is the most studied and the most frequently used barbiturate. It reduces cerebral blood flow and the cerebral metabolic rate, resulting in decreased ICP. Furthermore, it may have a protective effect on brain tissue during periods of hypoxia or hypoperfusion [19]. Barbiturates were used in $68.6 \%$ of the patients in this study. In cases of severe TBI, barbiturates may be administered in addition to hyperosmolar treatment.

A decompressive craniectomy is the surgical removal of a part of the skull in order to reduce ICP. This procedure may be combined with the evacuation of a mass lesion, such as a subdural hematoma, or as primary treatment for increased ICP. A surgeon may elect to perform a decompressive craniectomy if there is a rapid deterioration of clinical status. However, use of this technique in cases of severe TBI is 
controversial and its effectiveness is uncertain [20]. In our study, we performed a decompressive craniectomy on only 1 patient.

In conclusion, hyperosmolar treatment is routinely used for a wide range of acute brain injuries, including head trauma, ischemic stroke, and intracerebral bleeding. In this study, $3 \%$ hypertonic saline was used alone and in combination with mannitol. When compared with mannitol, fewer side effects were seen in the patients who received 3\% hypertonic saline. Our findings suggest that in planning hyperosmolar treatment in cases of TBI, especially in young children, 3\% hypertonic saline is safer and mannitol should be used carefully. However, there is a need for large-scale studies concerning the effectiveness and spectrum of side effects of osmotic treatments, especially in young children.

Ethics Committee Approval: The approval of the local Ethics Committee was obtained.

Peer-review: Externally peer-reviewed.

Authorship Contributions: Concept: A.Y.; Design: A.Y., F.A.; Data Collection or Processing: E.T.; Analysis or Interpretation: A.Y., E.T.; Literature Search: A.Y.; Writing: A.Y., E.T., F.A.

Conflict of Interest: None declared.

Financial Disclosure: The authors declared that this study received no financial support.

\section{References}

1. Corrigan JD, Selassie AW, Orman JA. The epidemiology of traumatic brain injury. J Head Trauma Rehabil 2010;25:72-80.

2. Karamanos E, Teixeira PG, Sivrikoz E, Varga S, Chouliaras K, Okoye $\mathrm{O}$, et al. Intracranial pressure versus cerebral perfusion pressure as a marker of outcomes in severe head injury: a prospective evaluation. Am J Surg 2014;208:363-71. [CrossRef]

3. Stein PD, Sabbah HN, Viano DC, Vostal JJ. Response of the heart to nonpenetrating cardiac trauma. J Trauma 1982;22:364-73.

4. Dewan MC, Mummareddy N, Wellons JC 3rd, Bonfield CM. Epidemiology of Global Pediatric Traumatic Brain Injury: Qualitative Review. World Neurosurg 2016;91:497-509.e1. [CrossRef]

5. Langlois JA, Rutland-Brown W, Thomas KE. Traumatic brain injury in the United States: emergency department visits, hospitalizations, and deaths. Available at: https://stacks.cdc.gov/ view/cdc/12294. Accessed Oct 2017.

6. Salottolo K, Levy AS, Slone DS, Mains CW, Bar-Or D. The effect of age on Glasgow Coma Scale score in patients with traumat- ic brain injury. JAMA Surg 2014;149:727-34. [CrossRef]

7. Dunning J, Daly JP, Malhotra R, Stratford-Smith P, Lomas JP, Lecky F, et al. The implications of NICE guidelines on the management of children presenting with head injury. Arch Dis Child 2004;89:763-7. [CrossRef]

8. Nakayama DK, Copes WS, Sacco WJ. The effect of patient age upon survival in pediatric trauma. J Trauma 1991;31:1521-6.

9. Ruppel RA, Kochanek PM, Adelson PD, Rose ME, Wisniewski SR, Bell MJ, et al. Excitatory amino acid concentrations in ventricular cerebrospinal fluid after severe traumatic brain injury in infants and children: the role of child abuse. J Pediatr 2001;138:18-25. [CrossRef]

10. Vavilala MS, Bowen A, Lam AM, Uffman JC, Powell J, Winn HR, et al. Blood pressure and outcome after severe pediatric traumatic brain injury. J Trauma 2003;55:1039-44. [CrossRef]

11. Jenkins LW, Kochanek PM. Developmental Neurobiology, Neurophysiology, and the PICU. In: Nichols DG, Shaffner DH, editors. Rogers'Textbook of Pediatric Intensive Care. Philadelphia: Lippincott Williams \& Wilkins; 2015. p. 861.

12. Stevens RD, Shoykhet M, Cadena R. Emergency Neurological Life Support: Intracranial Hypertension and Herniation. Neurocrit Care 2015;23 Suppl 2:S76-82. [CrossRef]

13. Xu W, Gerety P, Aleman T, Swanson J, Taylor J. Noninvasive methods of detecting increased intracranial pressure. Childs Nerv Syst 2016;32:1371-86. [CrossRef]

14. Favre JB, Ravussin P, Chiolero R, Bissonnette B. Hypertonic solutions and intracranial pressure. Schweiz Med Wochenschr 1996;126:1635-43.

15. Tasker RC, Acerini CL. Cerebral edema in children with diabetic ketoacidosis: vasogenic rather than cellular? Pediatr Diabetes 2014;15:261-70. [CrossRef]

16. Kochanek PM, Carney N, Adelson PD, Ashwal S, Bell MJ, Bratton $S$, et al. Guidelines for the acute medical management of severe traumatic brain injury in infants, children, and adolescents-second edition. Pediatr Crit Care Med 2012;13 Suppl 1:S1-82. [CrossRef]

17. Brophy GM, Human T, Shutter L. Emergency Neurological Life Support: Pharmacotherapy. Neurocrit Care 2015;23 Suppl 2:S48-68. [CrossRef]

18. Gantner D, Moore EM, Cooper DJ. Intravenous fluids in traumatic brain injury: what's the solution? Curr Opin Crit Care 2014;20:385-9. [CrossRef]

19. Cormio M, Gopinath SP, Valadka A, Robertson CS. Cerebral hemodynamic effects of pentobarbital coma in head-injured patients. J Neurotrauma 1999;16:927-36. [CrossRef]

20. Cooper DJ, Rosenfeld JV, Murray L, Arabi YM, Davies AR, D'Urso $\mathrm{P}$, et al. Decompressive craniectomy in diffuse traumatic brain injury. N Engl J Med 2011;364:1493-502. [CrossRef] 